\title{
Risk of heart failure after community acquired pneumonia: prospective controlled study with 10 years of follow-up
}

\author{
OPEN ACCESS
}

\author{
Dean T Eurich professor ${ }^{1}$, Thomas J Marrie professor ${ }^{3}$, Jasjeet K Minhas-Sandhu research \\ associate $^{2}$, Sumit R Majumdar professor ${ }^{124}$
}

'School of Public Health, University of Alberta, Edmonton, Alberta, Canada; ${ }^{2}$ ACHORD, 2-040 Li Ka Shing Center, University of Alberta, Edmonton, Alberta, Canada, T6G 2E1; ${ }^{3}$ Department of Medicine, Faculty of Medicine, Dalhousie University, Halifax, Nova Scotia, Canada; ${ }^{4}$ Department of Medicine, Faculty of Medicine and Dentistry, University of Alberta, Edmonton, Alberta, Canada

\begin{abstract}
Abstract

Objective - To determine the attributable risk of community acquired pneumonia on incidence of heart failure throughout the age range of affected patients and severity of the infection.

Design — Cohort study.

Setting - Six hospitals and seven emergency departments in Edmonton, Alberta, Canada, 2000-02.

Participants -4988 adults with community acquired pneumonia and no history of heart failure were prospectively recruited and matched on age, sex, and setting of treatment (inpatient or outpatient) with up to five adults without pneumonia (controls) or prevalent heart failure $(n=23$ 060).
\end{abstract}

Main outcome measures - Risk of hospital admission for incident heart failure or a combined endpoint of heart failure or death up to 2012, evaluated using multivariable Cox proportional hazards analyses.

Results - The average age of participants was 55 years, 2649 (53.1\%) were men, and $63.4 \%$ were managed as outpatients. Over a median of 9.9 years (interquartile range $5.9-10.6), 11.9 \%(n=592)$ of patients with pneumonia had incident heart failure compared with $7.4 \%(n=1712)$ of controls (adjusted hazard ratio $1.61,95 \%$ confidence interval 1.44 to 1.81). Patients with pneumonia aged 65 or less had the lowest absolute increase (but greatest relative risk) of heart failure compared with controls (4.8\% v 2.2\%; adjusted hazard ratio 1.98, 95\% confidence interval 1.5 to 2.53 ), whereas patients with pneumonia aged more than 65 years had the highest absolute increase (but lowest relative risk) of heart failure (24.8\% v18.9\%; adjusted hazard ratio $1.55,1.36$ to 1.77 ). Results were consistent in the short term (90 days) and intermediate term (one year) and whether patients were treated in hospital or as outpatients.

Conclusion - Our results show that community acquired pneumonia substantially increases the risk of heart failure across the age and severity range of cases. This should be considered when formulating post-discharge care plans and preventive strategies, and assessing downstream episodes of dyspnoea.

\section{Introduction}

Community acquired pneumonia is a common condition associated with substantial morbidity and mortality, irrespective of age. ${ }^{1}$ Community acquired pneumonia remains the seventh leading cause of death in the United States and accounts for an estimated \$9bn (£7bn; €8bn) in direct healthcare costs and 600 000 admissions to hospital each year. ${ }^{2-4}$ Although community acquired pneumonia is often considered an acute event, the long term associated risks are substantial ( $>50 \%$ mortality over five years and a nearly twofold higher risk of subsequent pneumonia than in controls without pneumonia). ${ }^{1}$ Moreover, despite major advances in risk stratification and management, downstream sequelae in survivors of pneumonia have not substantively improved over the past decade. ${ }^{5}$

Attention is increasingly focused on the link between pneumonia and subsequent cardiovascular diseases. Several studies have reported a clinically significant short term risk of major cardiovascular disease after acute respiratory tract infections, although the longer term impact of pneumonia on cardiovascular disease is less certain.$^{67}$ Heart failure is one cardiovascular complication that seems to be highly prevalent in patients with community acquired pneumonia. ${ }^{8-28}$ The mechanism by which pneumonia may influence heart failure is multidimensional. Acute infections can result in reduced myocardial function (eg, septic shock), increased oxygen consumption, tachycardia, and circulatory problems, which may all lead to increased cardiac workload and risk of heart failure. ${ }^{29-31}$

Overall, studies have reported incident or worsening heart failure rates of $1.4 \%$ in outpatient populations with community acquired pneumonia to as high as $24 \%$ among inpatients with 
pneumonia. ${ }^{7-28}$ Key study limitations have been the inability to distinguish between new onset heart failure and worsening heart failure, ${ }^{6-28}$ highly selected samples (cohorts derived from trial participants, patients admitted to hospital from a single medical centre, or elderly patients only), ${ }^{6-28}$ potential misclassification of patients with community acquired pneumonia (diagnosis based solely on administrative discharge coding) ${ }^{21-25}$ small sample sizes (<500 participants), ${ }^{8-28}$ short follow-up periods (eg, 30 day, or in-hospital events only), ${ }^{6-28}$ and relatively small number of events. ${ }^{8-28}$ Moreover, most studies have only focused on patients admitted to hospital ${ }^{8-28}$ and only one study has been able to distinguish the true attributable risk associated with pneumonia on heart failure events compared with a control population without pneumonia or prevalent heart failure; however, this study is limited as controls were not required to be selected from the same site of care (ie, risk estimates for patients with pneumonia admitted to hospital were calculated relative to patients without pneumonia who might not have been admitted) and this study was unable to account for any heart failure events occurring within 30 days of admission for pneumonia. $^{25}$

Thus it remains unclear if community acquired pneumonia truly increases the risk of incident heart failure in patients relative to those without pneumonia in the short term or longer term and whether the risk extends throughout the range of age and severity of community acquired pneumonia. We therefore assembled a representative population based cohort of patients with pneumonia and age-sex matched population controls without pneumonia, to better understand the clinical course of an episode of pneumonia and its comparative impact on short term and long term incidence of heart failure.

\section{Methods}

\section{Patients with pneumonia}

This prospective population based clinical registry has been extensively described in previous publications. ${ }^{1}$ All patients aged more than 17 years with community acquired pneumonia admitted during 2000-02 to any of the six hospitals or seven emergency departments in Edmonton, Alberta, Canada, which serves a population of approximately one million people, were enrolled in a clinical registry. We excluded patients who had tuberculosis or cystic fibrosis, were immunocompromised (eg, required $>10 \mathrm{mg} /$ day prednisone equivalents), or were pregnant. All patients with pneumonia had to have radiographic confirmation by treating clinicians and have at least two signs or symptoms (cough, pleuritic chest pain, shortness of breath, temperature $>38^{\circ} \mathrm{C}$, and crackles or bronchial breath sounds). All patients were treated according to a validated clinical pathway for community acquired pneumonia, which outlined preferred processes of care. ${ }^{32}$ Trained research nurses collected data prospectively from presentation to discharge using standardised abstract forms. These data included demographic information, doctor confirmed clinical and laboratory data, and use of prescription drugs within the week before admission.

\section{Matched controls}

We frequency matched each patient with pneumonia with up to five controls for age (five year age bands) and sex. Controls were required to be alive at the time of the pneumonia event (cases), presenting at the same site of care (same hospital for inpatients or same emergency department for outpatients) as the patient with pneumonia within the same month and year for a non-pneumonia related diagnosis, and have no history of community acquired pneumonia in the previous year based on
ICD-9-CM (international classification of diseases, nine revision, clinical modification) codes 480-487 or ICD-10 (international classification of diseases, 10th revision) codes J10-J18.

\section{Outcomes}

The primary outcome of interest was incident heart failure, which we defined as any heart failure related admission to hospital after the initial pneumonia event (or matched control index event) through to 31 March 2012. Hospital admissions with any diagnostic code for heart failure (ie, primary or any secondary diagnostic codes) were identified using the ICD-9-CM 428.x and ICD-10-CM I50 codes and ascertained by linking patients to comprehensive provincial healthcare administrative databases and vital statistics file. ${ }^{33} 34$ The quality and validity of the databases are routinely checked both provincially and federally with processes to resolve data issues where identified. Secondly, we evaluated the risk of incident heart failure in the short term (within 90 days of discharge after the index event) and intermediate term (within one year of discharge after the index event). We also evaluated a composite endpoint of incident heart failure or all cause mortality over the follow-up period.

\section{Statistical analysis}

As we were most interested in new onset heart failure after discharge, we excluded all patients with prevalent heart failure defined as any ICD-9-DM or ICD-10-CM codes for heart failure occurring before or during their initial pneumonia or matched control visit (minimum of three years before). Furthermore, we restricted our cohort to only those patients and controls who survived their initial index hospital admission or emergency department visit. We used Kaplan-Meier curves to describe the time to heart failure related hospital admission from the index pneumonia event (or matched control event).

To evaluate the outcomes for patients with pneumonia compared with controls we used multivariable Cox proportional hazard regression to account for the matched nature of these data. These data are presented as hazard ratios and $95 \%$ confidence intervals. No violations of proportional hazards assumptions were found through log-log plots and Schoenfeld residuals. Censoring occurred at departure from Alberta Health or 31 March 2012, providing a maximum follow-up of about 11 years. We did not consider repeat events; only time to first event was evaluated.

To ensure differences in outcomes were not driven by differences in comorbidity, we adjusted all analyses using the 31 coexisting conditions as defined by the Elixhauser comorbidity index. We calculated the Elixhauser index conditions based on diagnostic codes within the three years before the index event for patients with pneumonia and matched controls. Adjustments were also made for socioeconomic status (based on government level of support provided) and Aboriginal status (ie, First Nations and Inuit peoples), as well as the number of physician visits in the one year before the index event as an additional marker of disease burden. We also included age in the models to control for any residual confounding not accounted for in the matching process. In addition, to ensure results were consistent among inpatients and outpatients we stratified all analyses by site of care (inpatient and outpatient) for initial visit. Finally, as there are currently no estimates of heart failure risk in younger adults with community acquired pneumonia, we undertook additional analyses whereby we stratified our analyses into those aged less than 65 years and those aged more than 65 years to better understand the risk of heart failure associated with pneumonia throughout the age range. All analyses were conducted using SAS V9.4 (SAS Institute). 


\section{Sensitivity analysis}

Firstly, as the primary analysis excluded patients with new onset heart failure during their initial visit for the index event, we expanded the endpoint to include heart failure events that occurred within the initial visit for patients with pneumonia or matched control. Thus, the endpoint considered any patient with a diagnosis of incident heart failure during the index pneumonia event or any subsequent heart failure related hospital admission as having incident heart failure. Secondly, to better evaluate the risk of heart failure over the intermediate to long term, we excluded all patients admitted to hospital for heart failure within the first year after discharge, and repeated our analyses. Thirdly, we expanded our outcome definition to include not only heart failure related hospital admissions after discharge but also heart failure related emergency department visits. For this analysis, we only considered time to first emergency department visit or hospital admission for heart failure. Fourthly, to ensure incident heart failure is specific to pneumonia and not a marker of advanced illness or frailty, we repeated our analyses evaluating the risk of pneumonia on hospital admissions for stroke or fractures. We chose these conditions as they represent other major cardiovascular disease and difficult-to-measure frailty, respectively. Fifthly, as advanced diagnostic tests for heart failure were not routinely available at the time the cohort was established (eg, B type natriuretic peptide), we further excluded all users of diuretics as a prototypical marker of established heart failure to ensure prevalent heart failure could not be affecting our results. ${ }^{35}$ Sixthly, given the relatively high rates of mortality in this older population, we re-ran our primary analyses using a competing risk framework. ${ }^{36}$ Finally, we specifically evaluated the risk of new onset heart failure in patients with and without Streptococcus pneumoniae bacteraemia. $^{16}$

\section{Patient involvement}

No patients were involved in setting the research question or the outcome measures, nor were they involved in developing plans for recruitment, design, or implementation of the study. No patients were asked to advise on interpretation or writing up of results. There are no plans to disseminate the results of the research to study participants or the relevant patient community.

\section{Results}

Of the 6874 patients with community acquired pneumonia in the cohort, 314 (4.6\%) died in hospital, 857 (12.7\%) had prevalent heart failure, and $309(4.5 \%)$ had a heart failure code occurring within their initial pneumonia related visit and were excluded from our primary analyses. Of the remaining 5394 patients, 4988 were successfully matched with at least one control ( $n=23060 ; 3676(73.7 \%)$ with five controls). Of the 406 unmatched patients with pneumonia, $22(0.4 \%)$ could not be linked to administrative databases and $384(7.1 \%)$ did not have suitable controls (see supplementary figure). Overall, these patients were similar on age $(\mathrm{P}=0.17)$, were slightly more likely to be men $(59.1 \% \vee 53.1 \%, \mathrm{P}=0.02)$, and had similar severity of pneumonia $(\mathrm{P}>0.05)$. The median follow-up was 9.9 years (interquartile range 5.9-10.6), with a maximum follow-up of 11.4 years. Patients with pneumonia were aged 55 (SD 20) years, with $1762(35.3 \%)$ aged more than 65 years, and most were treated on an outpatient basis $(63.4 \%)$. Although patients with pneumonia and controls were evenly distributed by sex $(\mathrm{P}=0.60)$, controls were slightly younger (53 $v 55$ years, $\mathrm{P}<0.001)$ and had fewer comorbidities $(\Downarrow)$.
The primary endpoint of incident heart failure occurred in 592 (11.9\%) patients with pneumonia compared with 1712 (7.4\%) controls $(\mathrm{P}<0.001)$, translating into heart failure rates of 1.7 per 100 person years compared with 0.9 per 100 person years, respectively $(\mathrm{P}<0.001)(\Downarrow)$. After adjustment, patients with pneumonia had a higher rate of incident heart failure compared with the controls (adjusted hazard ratio 1.61, 95\% confidence interval 1.44 to $1.81, \mathrm{P}<0.001, \Downarrow)$. Moreover, compared with controls, patients with pneumonia were also more likely to have an incident heart failure related admission to hospital within 90 days of discharge after the index event $(1.4 \% v 0.6 \%$; adjusted hazard ratio $1.52,1.08$ to $2.13, \mathrm{P}=0.015)$ and also a higher risk at one year $(3.3 \%$ v $1.4 \% ; 1.86,1.50$ to $2.32, \mathrm{P}<0.001)$. Results were also consistent when stratified by site of care (inpatient versus outpatient) $(\Downarrow)$.

Stratification by age ( $\leq 65$ years and $>65$ years) showed that the risk of heart failure extended throughout the age range. Over follow-up, patients with pneumonia aged 65 or less had the lowest absolute difference for incident heart failure compared with controls $(4.8 \% v 2.2 \%)$ but the highest relative risk compared with controls $(1.98,1.55$ to 2.53$)$; whereas patients with pneumonia aged more than 65 had the highest absolute difference compared with controls $(24.8 \% v 18.9 \%)$ but the lowest relative risk $(1.55,1.36$ to 1.77$)$ (see supplementary table 1). These trends were also observed among inpatients and outpatients. Regardless of age, however, inpatients with pneumonia were at substantially higher risk for heart failure, particularly younger patients (see supplementary table 1).

Over the entire follow-up period, 1917 (38.4\%) patients with pneumonia and 5509 (23.9\%) controls died from any cause $(\mathrm{P}<0.001)$; this translates into mortality of 5.2 per 100 person years versus 2.9 per 100 person years, respectively $(\mathrm{P}<0.001)$. A substantial number of both patients with pneumonia and controls experienced the composite endpoint of incident heart failure or all cause mortality, with 2035 (40.8\%) patients with pneumonia and $6041(26.2 \%)$ controls having the composite endpoint. After adjustment, patients with pneumonia were also more likely to experience the combined endpoint of incident heart failure or all cause mortality than the controls (adjusted hazard ratio $1.53,95 \%$ confidence interval 1.44 to 1.63 , $\mathrm{P}<0.001)(\Downarrow)$. Again, the increased risk of the composite endpoint was observed for both inpatients and outpatients compared with controls $(\Downarrow)$.

\section{Sensitivity analyses}

Sensitivity analyses were robust and showed similar results to the primary analyses $(\Downarrow)$. After expanding the endpoint to include incident heart failure occurring within the initial index visit, patients with pneumonia were still at an increased risk of incident heart failure related events compared with their matched controls (15.0\% v 8.9\%; adjusted hazard ratio 1.82, 95\% confidence interval 1.65 to $2.01, \mathrm{P}<0.001$ ). Secondly, analyses excluding patients with incident heart failure within the first year $(n=485)$ also showed the increased risk of heart failure in patients with pneumonia over the intermediate to long term ( $8.9 \%$ v 5.9\%; adjusted hazard ratio $1.44,1.28$ to 1.61 ). Thirdly, after including incident heart failure related visits to an emergency department into the outcome, patients with pneumonia were still more likely to have heart failure related events $(14.0 \%$ v 8.8\%; $1.62,1.45$ to $1.80, \mathrm{P}<0.001)$. Fourthly, analyses evaluating the risk of stroke $(1.5 \% v 1.3 \% ; 1.02,0.77$ to 1.35$)$ or fractures $(4.8 \% v 4.0 \% ; 1.14,0.96$ to 1.35$)$ after pneumonia did not show any association, suggesting our results are more specific to heart failure in itself as opposed to overall patient illness or frailty. Fifthly, after excluding 424 patients 
with pneumonia with any use of diuretics as a potential marker of prevalent heart failure, our results remained robust $(10.2 \%$ $v 6.8 \% ; 1.39,1.25$ to 1.55$)$. Sixthly, our results were similar when re-evaluated using a competing risk framework (adjusted hazard ratio $1.37,1.25$ to $1.51, \mathrm{P}<0.001)$. Finally, compared with their matched controls $(n=460)$, patients with pneumococcal bacteraemia $(\mathrm{n}=98)$ were at statistically significant risk of incident heart failure $(13.3 \% v 7.4 \% ; 2.4,1.08$ to 5.3$)$. However, among patients with non-pneumococcal bacteraemia community acquired pneumonia $(\mathrm{n}=64)$ no increased risk compared with controls was observed $(\mathrm{n}=286 ; 12.5 \% v 10.8 \% ; 0.89,0.32$ to 2.4).

\section{Discussion}

In this large, population based cohort study of patients with community acquired pneumonia, we have highlighted the substantial increased risk of incident heart failure after an episode of community acquired pneumonia. Our data suggest that the 10 year risk of developing incident heart failure after a pneumonia event is approximately $12 \%$. Furthermore, compared with age-sex matched controls, there was a more than $50 \%$ relative increase in the risk of incident heart failure. This increased risk of heart failure after a pneumonia related event is conferred relatively early after discharge (within 90 days) but persists over the long term as well.

Although the risk of heart failure in elderly patients with community acquired pneumonia has been previously assessed, in our study we examined the potential risk of heart failure associated with pneumonia in a younger population. Heart failure in elderly people is well recognised, and also confirmed in our data, but this risk was also observed in young adults with pneumonia is also important. Indeed, among young adults with pneumonia managed on an inpatient basis, the risk of heart failure was substantially higher than in patients without pneumonia (the controls), with more than a threefold increase in absolute and relative risk compared with controls (5.8\% absolute risk increase, adjusted hazard ratio 3.6). Furthermore, although the absolute risk increase was lower, younger adults with pneumonia managed on an outpatient basis were also at considerable risk, and from a relative perspective the risk was similar to that observed among elderly inpatients with pneumonia compared with controls. Thus, although our data suggest that the highest absolute rates of heart failure are observed in elderly people, it is noteworthy that the highest relative rates of pneumonia associated heart failure are observed in younger adults managed in both inpatient and outpatient settings.

\section{Comparison with other studies}

To our knowledge, this is the longest outcomes study of patients with community acquired pneumonia and heart failure reported to date. Three previous studies evaluated the longer term risk of heart failure after an episode of pneumonia. ${ }^{18-25}$ Although the study by Adamuz et al reported one year outcomes, only deaths related to heart failure were reported and only included nine such deaths, making comparisons difficult. In a highly select study evaluating the effect of clarithromycin on cardiovascular events, Schembri et al observed that $3 \%$ of patients with community acquired pneumonia developed new or worsening heart failure within one year. This is consistent with our results at one year where we also observed $3 \%$ of patients with pneumonia having a hospital admission for heart failure, although among inpatients our results were slightly higher, with $6 \%$ admitted to hospital for heart failure within one year. Our results are also consistent with those in the study by Corrales-Medina et al, whereby $3.4 \%, 5.5 \%$, and $31 \%$ of elderly patients admitted to hospital for pneumonia developed heart failure within 90 days, one year, and beyond five years. ${ }^{25}$ Among our elderly patients admitted to hospital, $26.3 \%$ developed heart failure over the longer term (more than one year), and our relative rate to controls is nearly identical to that in the study by Corrales-Medina et al (adjusted hazard ratio $1.77 v 2.0$ ). Furthermore, in our combined inpatient and outpatient group $3.3 \%$ and $7.4 \%$ developed heart failure within 90 days and one year, largely driven by inpatients with community acquired pneumonia, which is also consistent with previous reports. ${ }^{25}$

Our results are less consistent with a previous meta-analysis, which suggested an overall short term risk (ie, inhospital and within 30 days of admission) of $14.1 \%$ among inpatients with pneumonia. ${ }^{22}$ Indeed, by 90 days of discharge only $1.4 \%$ of our cohort developed heart failure (2.3\% among inpatients and 3.3\% among elderly inpatients) and only approximately $5 \%$ had heart failure when inhospital heart failure events were included. The discrepancy in results is likely fully explained by the fact that many studies included in the meta-analysis were conducted in highly select populations, often with small sample sizes and events, often included patients with prevalent heart failure where incident or "worsening" heart failure was considered as opposed to just incident heart failure events as we have reported, and adjudication of heart failure was often based on clinical criteria as opposed to administrative coding in our study. ${ }^{22}$ Importantly, the meta-analysis was not able to distinguish the true attributable risk associated with pneumonia on heart failure events compared with a control population over the long term and was composed mainly of patients admitted to hospital. Indeed, although controls also have substantial long term risk of heart failure, both our study and the study by Corrales-Medina et al, highlight the considerable relative increase in risk of heart failure conferred by community acquired pneumonia. ${ }^{25}$ Moreover, we have extended these findings to show that the risk of heart failure after pneumonia is not merely in elderly people; pneumonia also confers a substantial risk of heart failure in young patients as well as outpatients.

Our study also builds on the evidence for patients with pneumonia managed as outpatients. Only one previous study has evaluated the impact of pneumonia on heart failure in patients managed on an outpatient basis. In 907 outpatients, the Pneumonia Patient Outcomes Research Team (PORT) observed an incident rate of heart failure of $1.4 \%$ within 30 days. ${ }^{11}$ Conversely, within 90 days only $0.9 \%$ of outpatients had incident heart failure in our study (with $8.2 \%$ developing heart failure over the entire follow-up). Again, the PORT study was fairly restrictive in the spectrum of patients included in their study and, unlike our study, was not population based.

\section{Strengths and limitations of study}

The putative role of community acquired pneumonia on heart failure, and cardiac disease in general, is still evolving. Pneumonia increases systematic oxidative stress and inflammatory markers (eg, circulatory cytokines), in both the short term and the long term, leading to an increased risk of thrombogenesis, destabilisation of atherosclerotic plaques, and endothelial dysfunction, potentially leading to increased rates of ischaemic heart disease, atrial fibrillation, and reduced ventricular function. ${ }^{6-37}$ Furthermore, this increased host response to infection has been shown to occur even in those with less severe infections and to persistent long after a patient clinically recovers from the acute infection. ${ }^{31}$ Whether community acquired pneumonia truly causes heart failure in itself or whether 
heart failure is simply the endgame in the cardiac cascade triggered by acute pneumonia event is not fully elucidated. Other factors, which are common to both pneumonia and heart failure, are also likely at play, including advanced age, reduced renal function, and the presence of other major comorbidities (eg, atherosclerosis, diabetes, hypertension), and collectively are likely to contribute to the increased risk of heart failure in patients with pneumonia. ${ }^{6}$ A major limitation of our study is that we could not examine these potential mechanisms and causes of heart failure related to pneumonia.

Additional limitations are that our study did not include certain high risk patient groups, most notably those who are immunocompromised. Although this would not be expected to lead directly to heart failure, immunocompromise is associated with frailty and potentially sicker patients who may be at increased risk of complications and chronic diseases, including heart failure. Secondly, our diagnosis of incident heart failure was based on administrative data as opposed to clinical data. However, heart failure codes in administrative data have been shown to be highly valid, with high positive and negative predictive values compared with medical records. ${ }^{334}$ Importantly, in both our patients with pneumonia and our controls we used the same method to identify incident heart failure, and no differential bias would be expected. Thirdly, as our cohort comprised only patients with community acquired pneumonia, we are unable to explore the reverse relationship of whether or not heart failure predisposes patients to the development of pneumonia. Lastly, owing to the administrative coding, we were unable to assess the degree of severity of heart failure in either of our cohorts. Given our outcome of hospital admission for heart failure, it is possible that milder cases of heart failure (eg, New York Heart Association classes I and II) might not have been easily detected, which may underrepresent the true incidence of heart failure in our study; however, we did include emergency department visits for heart failure as a sensitivity analysis, which would capture milder events, and this did not change our results. Furthermore, the relative effects we report are unlikely to be affected as no differential detection bias of milder heart failure is expected. Moreover, we were unable to account for some clinical differences (eg, blood pressure) that might have influenced heart failure events between the groups. We did, however, adjust our models for a well known and validated comorbidity index that would have captured doctor diagnosed hypertension. Despite these limitations, our study has several major strengths, including the large population based sample, use of controls to fully evaluate the potential incremental risk of pneumonia on heart failure, long term follow-up data, and extension of the literature across the range of ages and severity of pneumonia.

\section{Conclusion}

Our results show that patients with community acquired pneumonia are at increased risk for the future development of heart failure. Although elderly patients are often considered at high risk for heart failure, our results extend this risk to younger adults who survive an episode of pneumonia who may not be typically seen as higher risk for heart failure. Whether pneumonia is simply a marker of a high risk population or contributes to the underlying mechanism for the development of cardiovascular diseases and heart failure is strongly debated. ${ }^{622}$ Regardless, our results suggest that post-discharge care plans by front line clinicians, in partnership with patients, aimed at preventive measures might be warranted, including active screening and primary prevention strategies targeted at modifiable risk factors for cardiovascular disease. Moreover, increased attention to prevention of pneumonia, as the first consideration, through the use of pneumococcal (and seasonal influenza) vaccinations is also justified, particularly for those patients at higher risk of both pneumonia and cardiovascular disease (eg, elderly people, smokers). Furthermore, it highlights the possibility for both patients and their doctors that a subsequent episode of shortness of breath or wheezing might not be related to another respiratory tract infection but instead to new onset heart failure.

What is already known on this topic
Community acquired pneumonia has been
associated with new onset heart failure in elderly
patients
The attributable risk of pneumonia on incidence
of heart failure throughout the age range and
severity of pneumonia in patients is, however,
not known, particularly over the long term
What this study adds
Community acquired pneumonia substantially
increases the risk of heart failure, especially in
young adults, who had the highest relative rates
of pneumonia associated heart failure regardless
of pneumonia severity
This finding should be considered when
formulating post-discharge care plans and
preventive strategies, and assessing
downstream episodes of dyspnoea

Contributors: DTE and JMS were responsible for the study design, statistical analysis, interpretation of data, and drafting the manuscript. All authors contributed to the study design, interpretation of data, and critical revision of the manuscript. All authors, external and internal, had full access to all of the data (including statistical reports and tables) in the study and can take responsibility for the integrity of the data and the accuracy of the data analysis. DTE is the guarantor.

Funding: DTE receives salary support through a Canada Research Chair Award from the Government of Canada. SRM holds the Endowed Chair in Patient Health Management from the Faculties of Medicine and Dentistry and Pharmacy and Pharmaceutical Sciences, University of Alberta. TJM has received grants-in-aid from Capital Health, and unrestricted grants from Abbott Canada, Pfizer Canada, and Janssen-Ortho Canada.

Competing interests: All authors have completed the ICMJE uniform disclosure form at www.icmje.org/coi_disclosure.pdf and declare: no support from any organisation for the submitted work; no financial relationships with any organisations that might have an interest in the submitted work in the previous three years; no other relationships or activities that could appear to have influenced the submitted work.

Ethical approval: This study was approved by the health research ethics board at the University of Alberta.

Data sharing: No additional data available.

Transparency: The lead author (DTE) affirms that the manuscript is an honest, accurate, and transparent account of the study being reported; that no important aspects of the study have been omitted; and that any discrepancies from the study as planned (and, if relevant, registered) have been explained.

1 Eurich DT, Marrie TJ, Minhas-Sandhu JK, Majumdar SR. Ten-Year Mortality after Community-acquired Pneumonia. A Prospective Cohort. Am J Respir Crit Care Med 2015;192:597-604. doi:10.1164/rccm.201501-01400C.26067221

2 Huang SS, Johnson KM, Ray GT. Healthcare utilization and cost of pneumococcal disease in the United States. Vaccine 2011;29:3398-412.

doi:10.1016/j.vaccine.2011.02.088.21397721

3 Marrie TJ, Wu L. Factors influencing in-hospital mortality in community-acquired pneumonia: a prospective study of patients not initially admitted to the ICU. Chest 2005;127:1260-70. doi:10.1378/chest.127.4.1260.15821203

4 McAlister FA, Majumdar SR, Blitz S, Rowe BH, Romney J, Marrie TJ. The relation between hyperglycemia and outcomes in 2,471 patients admitted to the hospital with 
community-acquired pneumonia. Diabetes Care 2005;28:810-5. doi:10.2337/diacare.28.4.81015793178

5 Wang $\mathrm{Y}$, Eldridge N, Metersky ML. National trends in patient safety for four common conditions, 2005-2011. N Engl J Med 2014;370:341-51. doi:10.1056/NEJMsa1300991.24450892

6 Corrales-Medina VF, Musher DM, Wells GA, Chirinos JA, Chen L, Fine MJ. Cardiac complications in patients with community-acquired pneumonia: incidence, timing, risk factors, and association with short-term mortality. Circulation 2012;125:773-81. doi:10.1161/CIRCULATIONAHA.111.040766.22219349

7 Corrales-Medina VF, Alvarez KN, Weissfeld LA. Association between hospitalization for pneumonia and subsequent risk of cardiovascular disease. JAMA 2015;313:264-74 doi:10.1001/jama.2014.18229.25602997

8 Cabré M, Serra-Prat M, Force L, Palomera E, Pallarés R. Functional status as a risk factor for mortality in very elderly patients with pneumonia. Med Clin (Barc) 2008;131:167-70. doi:10.1157/1312426218674485

9 Aliberti S, Ramirez JA. Cardiac diseases complicating community-acquired pneumonia. Curr Opin Infect Dis 2014;27:295-301. doi:10.1097/QCO.0000000000000055.24685550

10 Viasus D, Garcia-Vidal C, Manresa F, Dorca J, Gudiol F, Carratalà J. Risk stratification and prognosis of acute cardiac events in hospitalized adults with community-acquired pneumonia. J Infect 2013:66:27-33. doi:10.1016/j.jinf.2012.09.003.22981899

11 Fine MJ, Stone RA, Singer DE. Processes and outcomes of care for patients with community-acquired pneumonia: results from the Pneumonia Patient Outcomes Research Team (PORT) cohort study. Arch Intern Med 1999;159:970-80 doi:10.1001/archinte.159.9.97010326939

12 Marrie TJ, Durant H, Yates L. Community-acquired pneumonia requiring hospitalization: 5-year prospective study. Rev Infect Dis 1989;11:586-99. doi:10.1093/clinids/11.4.5862772465

13 Esposito AL. Community-acquired bacteremic pneumococcal pneumonia. Effect of age on manifestations and outcome. Arch Intern Med 1984;144:945-8. doi:10.1001/archinte.1984.003501700810166712411

14 Janssens JP, Gauthey L, Herrmann F, Tkatch L, Michel JP. Community-acquired pneumonia in older patients. J Am Geriatr Soc 1996;44:539-44. doi:10.1111/j.1532-5415.1996.tb01439.x8617902

15 Fernández-Sabé N, Carratalà J, Rosón B. Community-acquired pneumonia in very elderly patients: causative organisms, clinical characteristics, and outcomes. Medicine (Baltimore) 2003;82:159-69. doi:10.1097/01.md.0000076005.64510.87.12792302

16 Musher DM, Rueda AM, Kaka AS, Mapara SM. The association between pneumococcal pneumonia and acute cardiac events. Clin Infect Dis 2007;45:158-65. doi:10.1086/518849.17578773

17 Becker T, Moldoveanu A, Cukierman T, Gerstein HC. Clinical outcomes associated with the use of subcutaneous insulin-by-glucose sliding scales to manage hyperglycemia in hospitalized patients with pneumonia. Diabetes Res Clin Pract 2007;78:392-7. doi:10.1016/j.diabres.2007.05.003.17597248

18 Adamuz J, Viasus D, Jiménez-Martínez E. Incidence, timing and risk factors associated with 1 -year mortality after hospitalization for community-acquired pneumonia. $J$ Infect 2014;68:534-41. doi:10.1016/j.jinf.2014.02.006.24534605

19 Yende S, Angus DC, Ali IS. Influence of comorbid conditions on long-term mortality after pneumonia in older people. J Am Geriatr Soc 2007;55:518-25. doi:10.1111/j.1532-5415.2007.01100.x.17397429

20 Schembri S, Williamson PA, Short PM. Cardiovascular events after clarithromycin use in lower respiratory tract infections: analysis of two prospective cohort studies. BMJ 2013;346:f1235. doi:10.1136/bmj.f1235.23525864

21 Mortensen EM, Halm EA, Pugh MJ. Association of azithromycin with mortality and cardiovascular events among older patients hospitalized with pneumonia. JAMA 2014;311:2199-208. doi:10.1001/jama.2014.4304.24893087

22 Corrales-Medina VF, Suh KN, Rose G. Cardiac complications in patients with community-acquired pneumonia: a systematic review and meta-analysis of observational studies. PLoS Med 2011;8:e1001048. doi:10.1371/journal.pmed.1001048.21738449
23 Corrales-Medina VF, Taljaard M, Fine MJ. Risk stratification for cardiac complications in patients hospitalized for community-acquired pneumonia. Mayo Clin Proc 2014;89:60-8. doi:10.1016/j.mayocp.2013.09.015.24388023

24 Perry TW, Pugh MJ, Waterer GW. Incidence of cardiovascular events after hospital admission for pneumonia. Am J Med 2011;124:244-51 doi:10.1016/j.amjmed.2010.11.014.21396508

25 Corrales-Medina VF, Taljaard M, Yende S. Intermediate and long-term risk of new-onset heart failure after hospitalization for pneumonia in elderly adults. Am Heart $J$ 2015;170:306-12. doi:10.1016/j.ahj.2015.04.028.26299228

26 Díaz A, Alvarez M, Callejas C, Rosso R, Schnettler K, Saldías F. [Clinical picture and prognostic factors for severe community-acquired pneumonia in adults admitted to the intensive care unit]. Arch Bronconeumol 2005;41:20-6. doi:10.1016/S1579-2129(06)60390-X15676132

27 Menéndez R, Torres A, Zalacaín RNeumofail Group. Risk factors of treatment failure in community acquired pneumonia: implications for disease outcome. Thorax 2004;59:960-5. doi:10.1136/thx.2003.017756.15516472

28 Querol-Ribelles JM, Tenías JM, Querol-Borrás JM. Levofloxacin versus ceftriaxone plus clarithromycin in the treatment of adults with community-acquired pneumonia requiring hospitalization. Int J Antimicrob Agents 2005;25:75-83. doi:10.1016/.ijantimicag.2004.07.013.15620830

29 Corrales-Medina VF, Madjid M, Musher DM. Role of acute infection in triggering acute coronary syndromes. Lancet Infect Dis 2010;10:83-92. doi:10.1016/S1473-3099(09)70331-7.20113977

30 Bazaz R, Marriott HM, Francis SE, Dockrell DH. Mechanistic links between acute respiratory tract infections and acute coronary syndromes. J Infect 2013;66:1-17. doi:10.1016/j.jinf.2012.09.009.23046969

31 Yende S, D'Angelo G, Mayr FGenIMS Investigators. Elevated hemostasis markers after pneumonia increases one-year risk of all-cause and cardiovascular deaths. PLOS One 2011;6:e22847. doi:10.1371/journal.pone.0022847.21853050

32 Marrie TJ, Lau CY, Wheeler SL, Wong CJ, Vandervoort MK, Feagan BG. A controlled trial of a critical pathway for treatment of community-acquired pneumonia. CAPITAL Study Investigators. Community-Acquired Pneumonia Intervention Trial Assessing Levofloxacin JAMA 2000;283:749-55. doi:10.1001/jama.283.6.74910683053

33 Quan H, Li B, Saunders LDIMECCHI Investigators. Assessing validity of ICD-9-CM and ICD-10 administrative data in recording clinical conditions in a unique dually coded database. Health Serv Res 2008;43:1424-41. doi:10.1111/j.1475-6773.2007.00822.x18756617

34 So L, Evans D, Quan H. ICD-10 coding algorithms for defining comorbidities of acute myocardial infarction. BMC Health Serv Res 2006;6:161. doi:10.1186/1472-6963-6-161.17173686

35 Udris EM, Au DH, McDonell MB. Comparing methods to identify general internal medicine clinic patients with chronic heart failure. Am Heart J 2001;142:1003-9. doi:10.1067/mhj.2001.119130.11717604

36 Fine JP, Gray RJ. A proportional hazards model for the subdistribution of a competing risk. J Am Stat Assoc 1999:94:496-509doi:10.1080/01621459.1999.10474144.

37 Van Eeden S, Leipsic J, Paul Man SF, Sin DD. The relationship between lung inflammation and cardiovascular disease. Am J Respir Crit Care Med 2012;186:11-6. doi:10.1164/rccm.201203-0455PP.22538803

\section{Accepted: 11012017}

Published by the BMJ Publishing Group Limited. For permission to use (where not already granted under a licence) please go to http://group.bmj.com/group/rights-licensing/ permissions This is an Open Access article distributed in accordance with the Creative Commons Attribution Non Commercial (CC BY-NC 4.0) license, which permits others to distribute, remix, adapt, build upon this work non-commercially, and license their derivative works on different terms, provided the original work is properly cited and the use is non-commercial. See: http://creativecommons.org/licenses/by-nc/4.0/. 


\section{Tables}

Table 1| Baseline characteristics of patients with community acquired pneumonia and sex and age matched controls. Values are numbers (percentages) unless stated otherwise

\begin{tabular}{|c|c|c|c|}
\hline Characteristics & Controls $(n=23060)$ & Patients with pneumonia $(n=4988)$ & $P$ value \\
\hline Mean (SD) age (years) & $53(20)$ & $55(20)$ & $<0.001$ \\
\hline \multicolumn{4}{|l|}{ Age categories: } \\
\hline$<25$ & $1919(8.3)$ & $373(7.5)$ & \multirow[t]{5}{*}{$<0.001$} \\
\hline $26-45$ & $7403(32.1)$ & $1482(29.7)$ & \\
\hline $46-65$ & $6684(29.0)$ & $1371(27.5)$ & \\
\hline $66-80$ & $4901(21.3)$ & $1118(22.4)$ & \\
\hline$>80$ & $2153(9.3)$ & $644(12.9)$ & \\
\hline Men & $12152(52.7)$ & $2649(53.1)$ & \\
\hline Welfare & $1524(6.6)$ & $542(10.9)$ & $<0.001$ \\
\hline Aboriginal people & $807(3.5)$ & $320(6.4)$ & $<0.001$ \\
\hline \multicolumn{4}{|l|}{ Treatment setting: } \\
\hline Outpatient & $15092(65.5)$ & $3163(63.4)$ & \multirow[t]{2}{*}{1.00} \\
\hline Inpatient & 7968 (34.5) & 1825 (36.6) & \\
\hline Mean (SD) physician visits in 1 year before index pneumonia event & $11(12)$ & $12(14)$ & $<0.001$ \\
\hline Mean (SD) length of follow-up (years) & $8.3(4.0)$ & $7.4(4.0)$ & $<0.001$ \\
\hline Median (IQR) length of follow-up (years) & $10.0(5.9-10.6)$ & $9.6(6.7)$ & \\
\hline Mean (SD) Charlson index score & $0.46(1)$ & $0.73(2)$ & $<0.001$ \\
\hline \multicolumn{4}{|l|}{ Elixhauser index: } \\
\hline Cardiac arrhythmia & $921(4.0)$ & $309(6.2)$ & $<0.001$ \\
\hline Valvular disease & $289(1.3)$ & $82(1.6)$ & 0.03 \\
\hline Pulmonary circulation disorders & $108(0.5)$ & $73(1.5)$ & $<0.001$ \\
\hline Peripheral vascular disorders & $428(1.9)$ & $131(2.6)$ & $<0.001$ \\
\hline Uncomplicated hypertension & $2303(10.0)$ & $601(12.0)$ & $<0.001$ \\
\hline Other neurological disorders & $556(2.4)$ & $263(5.3)$ & $<0.001$ \\
\hline Chronic obstructive pulmonary disease & $1186(5.1)$ & $796(16.0)$ & $<0.001$ \\
\hline Uncomplicated diabetes & $892(3.9)$ & $275(5.5)$ & $<0.001$ \\
\hline Complicated diabetes & $269(1.2)$ & $80(1.6)$ & 0.01 \\
\hline Hypothyroidism & $559(2.4)$ & $194(3.9)$ & $<0.001$ \\
\hline Renal failure & $222(1.0)$ & $83(1.7)$ & $<0.001$ \\
\hline Liver disease & $347(1.5)$ & $150(3.0)$ & $<0.001$ \\
\hline Solid tumour without metastasis & $908(3.9)$ & $193(3.9)$ & 0.82 \\
\hline Rheumatoid arthritis & $234(1.0)$ & $96(1.9)$ & $<0.001$ \\
\hline Obesity & $495(2.1)$ & $156(3.1)$ & $<0.001$ \\
\hline Fluid and electrolyte disorders & $2096(9.1)$ & $629(12.6)$ & $<0.001$ \\
\hline Alcohol misuse & $816(3.5)$ & $324(6.5)$ & $<0.001$ \\
\hline Drug misuse & $551(2.4)$ & $225(4.5)$ & $<0.001$ \\
\hline Psychoses & $393(1.7)$ & $98(2.0)$ & 0.20 \\
\hline Depression & $1179(5.1)$ & $365(7.3)$ & $<0.001$ \\
\hline
\end{tabular}


Table 2| Risk of heart failure or heart failure and death among patients with community acquired pneumonia and controls. Values are numbers (percentages) unless stated otherwise

\begin{tabular}{|c|c|c|c|c|}
\hline Analyses & Controls $(n=23060)$ & Patients with pneumonia $(n=4988)$ & Adjusted hazard ratio $(95 \% \mathrm{Cl})$ & $P$ value \\
\hline \multicolumn{5}{|l|}{ Primary analysis } \\
\hline \multicolumn{5}{|l|}{ Incident heart failure: } \\
\hline Inpatients & $875(11.0)$ & $334(18.3)$ & 1.94 (1.64 to 2.29$)$ & $<0.001$ \\
\hline Outpatients & $837(5.6)$ & $258(8.2)$ & $1.33(1.12$ to 1.57$)$ & $<0.001$ \\
\hline \multicolumn{5}{|c|}{ Incident heart failure within 90 days: } \\
\hline Combined endpoint & $145(0.6)$ & $68(1.4)$ & 1.52 (1.08 to 2.13$)$ & $<0.001$ \\
\hline Inpatients & $80(1.0)$ & $41(2.3)$ & $1.45(0.90$ to 2.34$)$ & 0.1 \\
\hline Outpatients & $65(0.4)$ & $27(0.9)$ & 1.38 (0.79 to 2.39$)$ & 0.3 \\
\hline Outpatients & $140(0.9)$ & $58(1.8)$ & 1.54 (1.08 to 2.20$)$ & 0.019 \\
\hline \multicolumn{5}{|c|}{ Incident heart failure or all cause mortality: } \\
\hline Combined endpoint & $6041(26.2)$ & $2035(40.8)$ & $1.53(1.44$ to 1.63$)$ & $<0.001$ \\
\hline Inpatients & $3172(39.8)$ & $1178(64.5)$ & $1.77(1.62$ to 1.93$)$ & $<0.001$ \\
\hline Outpatients & $2869(19.0)$ & $857(27.1)$ & 1.34 (1.23 to 1.47$)$ & $<0.001$ \\
\hline
\end{tabular}




\section{Figures}

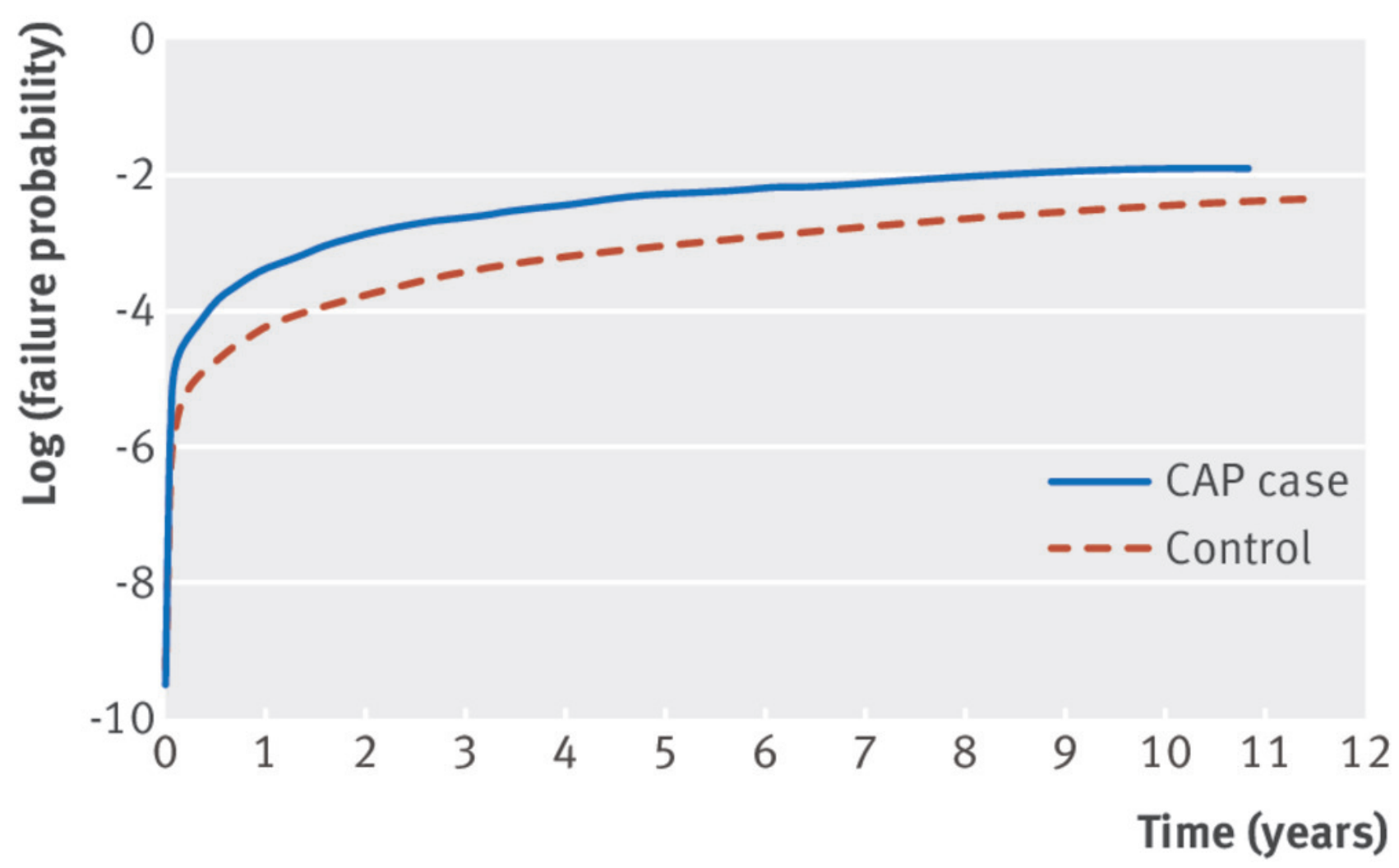

Cumulative incidence of heart failure. $\mathrm{CAP}=$ community acquired pneumonia

\section{Main analyses}

Incident heart failure (HF)

\section{Sensitivity analyses}

Inclusion of HF during index pneumonia event Exclusion of $\mathrm{HF}$ during first year Inclusion of emergency department HF related events Risk of stroke after pneumonia Risk of fractures after pneumonia Exclusion of patients using diuretics Competing risk analysis with death Pneumococcal bacteraemia Non-pneumococcal bacteraemia

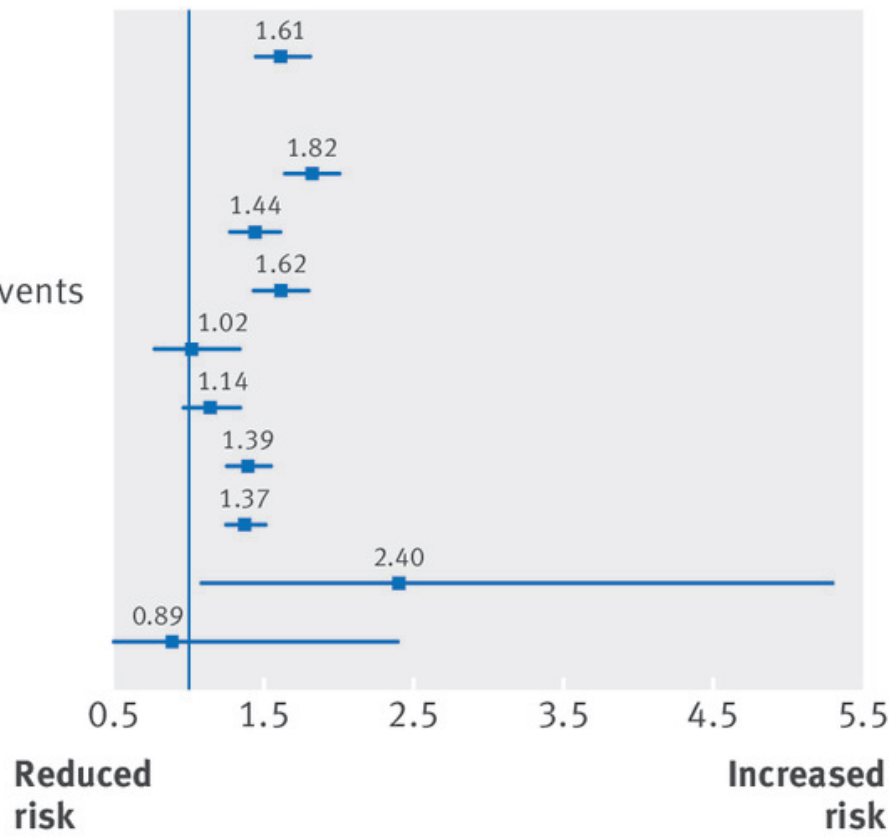

Adjusted hazard ratios for incident heart failure in patients with community acquired pneumonia compared with controls 